\title{
Resistencia a insulina en adolescentes obesos
}

\author{
Insulin resistance in obese adolescents
}

\author{
Maria Isabel Rojas-Gabulli1,2, Oswaldo Núñez ${ }^{1,2}$, Carlos Del Águila ${ }^{1,2}$, \\ Mariel Briceño ${ }^{1}$, Nelly Valenzuela ${ }^{2}$ \\ ${ }^{1}$ Servicio de Endocrinología, Instituto Nacional de Salud del Niño. Lima, Perú. \\ ${ }^{2}$ Facultad de Medicina, Universidad Nacional Federico Villarreal. Lima, Perú.
}

\begin{abstract}
Resumen
Introducción: La obesidad en niños y adolescentes es un problema de salud pública que se encuentra en incremento a nivel mundial, siendo conocida su relación con el desarrollo de alteraciones metabólicas como la diabetes mellitus tipo 2. Objetivos: Determinar la presencia de resistencia a la insulina en adolescentes obesos y no obesos según sexo, edad, estadio puberal y antecedentes familiares de alteración metabólica o enfermedad cardiovascular (ECV) explorando asociación y riesgo. Diseño: Estudio transversal, observacional y analítico. Lugar: Tres centros educativos de la zona urbana de Lima. Participantes: Adolescentes entre 10 y 17 años de edad. Intervención: Se estudió 69 adolescentes entre 10 y 17 años de edad, agrupados de acuerdo a la presencia de obesidad. El diagnóstico de obesidad fue definido por un índice de masa corporal (IMC) $>95$ percentil; $55,1 \%$ varones y $44,9 \%$ mujeres. Se empleó el modelo de evaluación homeostática para determinar la resistencia a insulina (HOMA-IR) y la función de la célula beta (HOMA-\%B) en ambos grupos. También se realizó determinaciones de lípidos mediante método enzimático y se aplicó una encuesta para buscar antecedentes familiares de obesidad, diabetes mellitus y enfermedad cardiovascular (ECV). El análisis estadístico se hizo mediante pruebas de correlación de Spearman y de comparación de medias con la prueba U de Mann- Whitney. Principales medidas de resultados: Insulina basal, colesterol total, triglicéridos, colesterol-VLDL, HOMA IR y HOMA\%B en adolescentes obesos y no obesos. Resultados: Se encontró que la insulina basal, el colesterol total, triglicéridos, colesterol-VLDL, HOMA IR y HOMA\%B fueron significativamente mayores $(p<0,05)$ en el grupo de obesos. El HOMA-IR presentó una correlación inversa, débil, con el desarrollo puberal, particularmente en el sexo femenino $(r=-0,486, p<0,05)$. Predominaron los antecedentes familiares de obesidad $y E C V$ en el grupo de obesos (71,8\%). Conclusiones: La presencia de resistencia a la insulina en adolescentes obesos se asocia a los antecedentes familiares de obesidad y ECV. Se sugiere el empleo del HOMA asociado a la identificación de dichos antecedentes en adolescentes obesos, a fin de incorporarlos a un programa de atención integral de orientación a un estilo de vida saludable.

Palabras clave: Obesidad; resistencia a insulina; salud pública.
\end{abstract}

\begin{abstract}
Introduction: Obesity in children and adolescents is a public health problem that is increasing worldwide, being known its relation with the development of metabolic alterations like diabetes mellitus type 2. Objectives: To determine the presence of insulin resistance in obese and non obese adolescents according to sex, age, pubertal stage and presence of familial metabolic or cardiovascular disorders. Design: Cross-sectional observational and analytical study. Setting: Three Lima urban educational centers. Participants: Adolescents 10 to 17 year-old. Interventions: Sixty nine adolescents between 10 and 17 years old were divided in two groups according to the presence of obesity. Obesity was defined as body mass index $>95$ th percentile, $55,1 \%$ were male and $44,9 \%$ female. Homeostatic model assessment was employed to determine insulin resistance (HOMA-IR) and beta cell function (HOMA\%B) in both groups. Lipid levels were measured by the enzymatic method and a questionnaire was applied to determine familial obesity, diabetes mellitus and cardiovascular disease. Spearman correlation and U Mann-Whitney for media comparison tests were employed. Main outcome measures: Basal insuline, total cholesterol, triglicerids, CLDL cholesterol, HOMA IR and HOMA\%B in obese and non obese adolescents. Results: Basal insulin, cholesterol, triglycerides, VLDL cholesterol, HOMA IR and HOMA\%B were significantly higher $(p<0,05)$ in the obese group. HOMA -IR showed a weak inverse correlation with Tanner stage in females. Cardiovascular disease and obesity predominated in the obese group families $(71,8 \%)$. Conclusions: The use of HOMA along with familial disorders identification in obese adolescents is recommended in order to incorporate them in a healthy lifestyle program.
\end{abstract}

Key words: Obesity; insulin resistance; public health.

\section{INTRODUCCIÓN}

La obesidad en niños y adolescentes es un problema de salud pública que se encuentra en incremento a nivel mundial; es conocida su relación con el desarrollo de alteraciones metabólicas, como la diabetes mellitus tipo 2 (DM2) ${ }^{(1)}$, y se refiere que esta última se presenta como una epidemia emergente en la edad pediátrica, con una mayor incidencia durante la adolescencia, etapa en la cual se aprecia también un aumento de la resistencia a la insulina ${ }^{(2)}$. Hugo Amigo, tomando datos de la OMS del año 2000, encontró un aumento de la obesidad en América
Latina, que incluyó al Perú dentro del grupo de aquellos que poseían valores altos de déficit de peso y aumento de obesidad en el rango de brote epidémico (3). En la ciudad de Lima, se ha hallado un alarmante $39,6 \%$ de prevalencia de sobrepeso y obesidad ${ }^{(4)}$.

Respecto a la composición corporal y factores metabólicos, algunos autores han encontrado en una muestra étnicamente diversa de niños obesos una relación compleja entre la distribución de la grasa, composición corporal y factores metabólicos y han señalado que, dado que la información en este sentido es limitada, es esencial lograr una mejor comprensión de factores, tales como la composición corporal y resistencia a insulina (RI) asociadas a esta epidemia (5). En nuestro medio, hasta el momento no se ha publicado estudios al respecto en la población infantil.

Diversos estudios han demostrado que la diabetes tipo 2 en adultos se desarrolla en un periodo largo y que la mayoría de pacientes presentan inicialmente intolerancia a la glucosa, estado intermedio en la historia natural de la DM2, que predice su desarrollo y el de la enfermedad cardiovascular ${ }^{(6-8)}$; mientras que 
otra investigación describió que con las recomendaciones apropiadas y cambios en el estilo de vida se puede evitar o por lo menos diferir el avance hacia la diabetes clínica ${ }^{(9)}$. Reaven y Ferranini, en distintos momentos, establecieron que la obesidad es la causa más común de resistencia a insulina e hiperinsulinemia en humanos y que contribuye al desarrollo de alteraciones cardiovasculares, hiperglicemia y dislipidemia, constituyendo el síndrome metabólico o síndrome $\mathrm{X}^{(10,11)}$. En un estudio en nuestro medio, se ha señalado que el principal problema del niño obeso es la hipertrigliceridemia y que pudiera ser un factor, independiente de la obesidad, que sumaría riesgo para problemas cardiovasculares en la vida adulta ${ }^{(12)}$.

La insulina es una de las tres hormonas peptídicas de la familia de factores de crecimiento semejantes a insulina (IGFs), junto con la IGF-I e IGF-II, siendo esencialmente reguladora de funciones metabólicas en el hígado, músculo y tejido adiposo, mientras que las otras dos son importantes para la función de casi todos los órganos en el cuerpo. El principal estímulo hormonal para la producción de IGF-I es la hormona de crecimiento (HC), pero el estado nutricional y el aporte energético y proteico en la dieta son también elementos críticos para su regulación y las de sus proteínas transportadoras ${ }^{(13-16)}$.

Estudios realizados en niños obesos púberes y prepúberes han demostrado que la hiperinsulinemia y la RI están bien establecidas, aún en las etapas iniciales de la obesidad, pero la influencia de dicha hiperinsulinemia sobre el eje HC-IGF-I y su relación con la pubertad temprana en mujeres aún no ha sido completamente aclarada ${ }^{(17)}$.

Rosenbloom señala que la obesidad severa en niños y adolescentes, tiene un rol prominente en la patogénesis de la DM2 ${ }^{(18)}$. Posteriormente, en un estudio transversal, Sinha y col encuentran que el índice de resistencia a insulina, determinado mediante el modelo de evaluación homeostática (HOMA-IR) propuesto por Matthews y col, predecía fuertemente el nivel de glicemia a las 2 horas posprandial, sugiriendo que el factor de riesgo más importante para el desarrollo de la alteración de la tolerancia a glucosa es la obesidad infantil severa ${ }^{(19,20)}$.

Por otra parte, un signo frecuentemente asociado a hiperinsulinemia y obesidad, la acantosis nigricans, se presenta con mayor frecuencia en poblaciones con mayor prevalencia de DM2, como las afroamericanas, hispanas y nativo americanas. Yanosvski propone que esta situación pudiera estar influenciada por los mayores niveles de IGF-I encontrados en los afroamericanos en relación a la población caucásica ${ }^{(21)}$.

Muchos factores pueden afectar potencialmente la tendencia a continuar la obesidad a lo largo de los años, y la RI ha sido sugerida como uno de esos factores. Sin embargo, algunos autores señalan que, aunque se ha demostrado la relación entre la RI y la ganancia de peso, no está claro si la RI es un factor que promueve o simplemente una consecuencia de la ganancia de peso ${ }^{(22)}$. En relación, a esto hay datos contrastantes entre adultos y niños.

Maffeis y col plantearon que la relación entre RI y ganancia de peso puede ser un proceso dinámico que se altera con la edad y concluyen, en un estudio de seguimiento de niños obesos después de 14 años, que el impacto de la RI en la niñez para predecir la obesidad en mujeres fue muy relevante y que el grado de resistencia a la insulina en niñas con sobrepeso, pero no en los niños, fue un factor protector (independiente) de obesidad en la vida adulta. Las razones son desconocidas y este hallazgo no fue afectado por el grado de obesidad en la infancia, edad, estadio puberal o IMC de los padres ${ }^{(23)}$.

El espectro de las situaciones anteriormente expuestas y la falta de estudios en nuestro medio constituyen una motivación para la evaluación de resistencia a la insulina. Esto es particularmente importante porque datos recientes sugieren que los efectos de la RI y los factores de riesgo de ECV pueden ser mitigados y que la DM2 puede ser prevenida mediante la intervención temprana en los individuos afectados. Además, podremos comparar nuestros resultados con lo expuesto en la literatura y determinar en nuestra pobla- ción de estudio los posibles indicadores de riesgo de continuar obesidad en la edad adulta, así como presentar alteraciones en el metabolismo de la glucosa.

Así, el objetivo del presente estudio fue determinar la presencia de resistencia a la insulina en adolescentes obesos y no obesos, según sexo, edad, estadio puberal y antecedentes familiares de alteración metabólica o enfermedad cardiovascular (ECV), explorando asociación y riesgo.

\section{MÉTODOS}

Se realizó un estudio transversal, observacional y analítico durante el segundo y tercer trimestres del año 2003, en tres centros educativos de una zona urbana en Lima. La población fue de 69 escolares, entre 10 y 17 años de edad, en quienes se obtuvo el consentimiento informado de los padres y se cumplió los criterios de inclusión; 34 fueron calificados como obesos y 35 -de características similarescomo no obesos. Se excluyó a los adolescentes que se encontraban recibiendo medicamentos o estaban en tratamiento para reducción de peso, a quienes tenían antecedente de dislipidemia primaria y a quienes padecían enfermedad crónica. Se entregó información escrita acerca del estudio a todos los padres, para obtener su consentimiento y el de los pacientes, así como una encuesta acerca de antecedentes familiares de diabetes, obesidad o enfermedad cardiovascular, por línea materna y paterna, hasta tercer grado de consanguinidad.

A todos ellos se les realizó una evaluación física y antropométrica para determinar su estado nutricional y etapa puberal, según Marshall y Tanner ${ }^{(24,25)}$, a cargo de los investigadores capacitados en técnicas antropométricas. Se calificó como obeso cuando el índice de masa corporal (IMC) fue $>95$ percentil de las tablas recomendadas por la Organización Mundial de la Salud, de acuerdo a los criterios de Must ${ }^{(26)}$. Adicionalmente se estableció el grado de obesidad severa cuando la desviación estándar fue mayor a 2,5 (similar al 97p).

La colección y procesamiento de muestras estuvo a cargo de una profesional de tecnología médica. Se extrajo 
una muestra de sangre venosa en posición sentada, luego de 12 horas de ayuno, y se procedió a la separación del suero por centrifugación a $5000 \mathrm{rpm}$ durante 10 minutos, para ser congelado en crioviales a $-20^{\circ} \mathrm{C}$, hasta su procesamiento. La determinación de glucosa, colesterol total, colesterol de lipoproteína de alta densidad (C-HDL) y triglicéridos (TGC) se realizó por método enzimático, en un lector espectrofotómetro marca Microlab 200 de Merck, en condiciones iguales de temperatura y tiempo.

Los valores de colesterol de lipoproteína de baja densidad (C-LDL) fueron obtenidos mediante la fórmula de Friedewald:

\section{$\mathrm{C}-\mathrm{LDL}=$ colesterol total $-\mathrm{HDL}$ colesterol - (TGC/5)}

Se consideró como valores normales los establecidos por el NCEP (1991):

Colesterol total (CT) : $<170 \mathrm{mg} / \mathrm{dL}(<90$ percentil)

Colesterol-lipoproteína de alta densidad (CHDL): $>35 \mathrm{mg} / \mathrm{dL}$ ( $>5$ percentil)

Colesterol-lipoproteína de baja densidad (CLDL): $<130 \mathrm{mg} / \mathrm{dL}$ (<90 percentil)

Triglicéridos (TGC): $<100 \mathrm{mg} / \mathrm{dL} \quad(<90$ percentil)

La dislipidemia fue definida como la presencia de valores de lípidos fuera de los rangos de normalidad anteriormente descritos, los cuales fueron verificados inmediatamente en las mismas condiciones y bajo la misma metodología.

Para la determinación de insulina se empleó el método de ELISA en un equipo EL 301 BIOTEK.

La resistencia a la insulina se determinó aplicando el modelo de evaluación homeostática de la sensibilidad a la insulina (HOMA-IR), según lo descrito por Matthews ${ }^{(16)}$, con la siguiente fórmula:

$$
\begin{gathered}
\text { HOMA-IR }=(\text { insulina basal }(\mathrm{UU} / \mathrm{mL}) \mathrm{x} \\
\text { glicemia basal }(\mathrm{mmol} / \mathrm{L})) / 22,5
\end{gathered}
$$

Los resultados de HOMA-IR fueron agrupados en 3 categorías, según percentiles (grupo $1<33$; grupo $233-66$ y grupo $3>66 \mathrm{p}$ ), a fin de observar si a mayor
HOMA-IR existía mayor asociación con la presencia de obesidad.

También se evaluó la función de la célula beta mediante la siguiente ecuación:

\section{HOMA-\%B $=(20 \mathrm{x}$ insulina basal $(\mathrm{uU} / \mathrm{mL})) /$ (glicemia basal (mmol/L) -3,5)}

HOMA-\%B (\% beta-secreción). El Homa-2 es una actualización y adaptación hecha por Jonathan Levy y col. en Oxford, la cual establece una relación no lineal entre glucosa e insulina (que se ajusta mejor a la realidad fisiológica). Toma en cuenta las variaciones en la resistencia hepática y en la tisular periférica. En adultos, considera aumentos en la curva de secreción de insulina cuando las glucemias superan $180 \mathrm{mg} /$ dL. Sin embargo, al no existir rangos de normalidad estadística en adolescentes para el presente estudio, el HOMA-\%B fue aplicado en $100 \%$ y se evaluó las diferencias entre el grupo de obesos y no obesos.
Todos lo datos obtenidos fueron registrados en una base de datos y analizados con la ayuda del paquete estadístico SPSS versión 10,0. Una vez realizado el análisis descriptivo de las variables de estudio, se hizo pruebas de correlación de Spearman y de comparación de medias, mediante la prueba U de Mann- Whitney. Para medir asociación entre variables se utilizó la prueba chi cuadrado. Para establecer el riesgo, se calculó el odds ratio. Se consideró significativos valores de $p<0,05$.

\section{RESULTADOS}

Se evaluó un total de 69 escolares adolescentes: 34 con obesidad $(49,2 \%)$ y 35 no obesos $(50,7 \%)$; la distribución por sexos se observa en la tabla 1 . Hubo un total de 19 escolares prepúberes $(27,5 \%)$, nueve de los cuales fueron obesos y 10 no obesos.

En la tabla 2 se muestra algunas de las características evaluadas en ambos grupos de estudio. Según se puede apreciar, los

\begin{tabular}{|c|c|c|c|c|c|c|}
\hline \multirow[t]{2}{*}{ Sexo } & \multicolumn{2}{|c|}{ Obesos } & \multicolumn{2}{|c|}{ No obesos } & \multicolumn{2}{|c|}{ Total } \\
\hline & $n$ & $\%$ & $\mathrm{n}$ & $\%$ & $n$ & $\%$ \\
\hline Masculino & 18 & 52,9 & 20 & 57,1 & 38 & 55,1 \\
\hline Femenino & 16 & 47,1 & 15 & 42,9 & 31 & 44,9 \\
\hline
\end{tabular}

Tabla 1. Escolares por sexo y según estado nutricional.

\begin{tabular}{|c|c|c|c|c|c|}
\hline \multirow[t]{2}{*}{ Características } & \multicolumn{2}{|c|}{ Obesos } & \multicolumn{2}{|c|}{ No obesos } & \multirow[t]{2}{*}{$p$} \\
\hline & Media & DS & Media & DS & \\
\hline Edad (años) & 11,50 & 1,36 & 12,5 & 2,4 & 0,221 \\
\hline Peso al nacer (g) & 3389,33 & 452,9 & 3454,52 & 535,0 & 0,465 \\
\hline IMC & 25,6 & 2,3 & 18,2 & 1,7 & $0,001^{*}$ \\
\hline Glicemia basal (mg/dL) & 79,7 & 6,8 & 76,3 & 9,6 & 0,130 \\
\hline Insulina basal (uU/mL) & 9,8 & 13,1 & 4,3 & 3,8 & $0,004^{*}$ \\
\hline HOMA-IR & $\begin{array}{c}1,89 \\
(0,25-12,45)\end{array}$ & 2,36 & $\begin{array}{c}0,82 \\
(0,19-4,26)\end{array}$ & 0,8 & $0,003^{*}$ \\
\hline HOMA-\%B & 292,53 & 558,92 & 94,5 & 152,3 & $0,014^{*}$ \\
\hline Colesterol (mg/dL) & 184,2 & 39,9 & 146,3 & 41,7 & $0,028^{*}$ \\
\hline LDL- colesterol (mg/dL) & 100,4 & 36,8 & 90,3 & 39,2 & 0,0065 \\
\hline HDL colesterol (mg/dL) & 35,1 & 5,7 & 36,3 & 6,4 & 0,592 \\
\hline Triglicéridos (mg/dL) & 143,5 & 66,0 & 98,9 & 27,9 & $0,001^{*}$ \\
\hline C-VLDL (mg/dL) & 28,7 & 13,2 & 19,8 & 5,6 & $0,001^{*}$ \\
\hline
\end{tabular}

Tabla 2. Características de los grupos de estudio.

${ }^{*} p<0,05$. 
valores de insulina basal, colesterol total, triglicéridos y C-VLDL fueron significativamente mayores en el grupo de obesos y los indicadores de resistencia a insulina y función de células beta (HOMA-IR y HOMA-\%B ) fueron igualmente significativos $(p<0,05)$. También, se encontró que $43 \%$ de los adolescentes obesos presentó dislipidemia secundaria (hipercolesterolemia, HDL-colesterol disminuido o hipertrigliceridemia o una combinación entre ellas), mientras que solo se observó esta alteración en 23,6\% del grupo sin obesidad.

Al analizar los indicadores de resistencia a la insulina en relación al estadio de desarrollo puberal, se observó que la insulina y el HOMA fueron notoriamente mayores en los estadios 2 y 4 (tabla 3 ). El HOMA y la insulina se correlacionaron de manera inversa, aunque débil, con los estadios de Tanner (HOMA, p=0,005, $\mathrm{r}=-0,336$ e insulina $p=0,012, \mathrm{r}=-0,300)$. No se halló relación con la edad cronológica.

En la tabla 4 se puede ver que en el grupo de obesos predominó la categoría 3 del HOMA, correspondiente a los valores por encima del 66 percentil, lo cual también obtuvo una diferencia estadísticamente significativa.

En cuanto a los antecedentes familiares, se encontró que $71,8 \%$ de los obesos presentó antecedentes de obesidad o enfermedad cardiovascular y solo $28,2 \%$ en el grupo de los no obesos. Al analizarse estos antecedentes con el HOMA-IR y el HOMA-\%B no se observó ninguna relación. Sin embargo, al evaluar la relación antecedentes familiares de riesgo de enfermedad cardiovascular y obesidad, se encontró que era significativamente mayor en el grupo con obesidad (tabla 5).

Tabla 3. Insulina y resistencia a insulina según estadio puberal (Tanner).

\begin{tabular}{|c|c|c|c|c|c|c|}
\hline \multirow{2}{*}{$\begin{array}{l}\text { Estadio puberal } \\
\qquad \text { (Tanner) }\end{array}$} & \multicolumn{2}{|c|}{ Insulina } & \multicolumn{2}{|c|}{ HOMA-IR } & \multicolumn{2}{|c|}{ HOMA-\%B } \\
\hline & Media & DS & Media & DS & Media & DS \\
\hline 1 & 6,6 & 4,4 & 1,3 & 0,8 & 190,1 & 228,2 \\
\hline 2 & 10,4 & 16,2 & 1,9 & 2,8 & 319,2 & 704,2 \\
\hline 3 & 3,5 & 3,1 & 0,7 & 0,7 & 104,5 & 92,7 \\
\hline 4 & 9,9 & 12,0 & 1,9 & 2,4 & 181,1 & 384,9 \\
\hline 5 & 4,4 & 5,9 & 0,8 & 1,2 & 111,5 & 152,3 \\
\hline
\end{tabular}

Tabla 4. Grupos de estudio según categorías de resistencia a insulina (HOMA-IR).

\begin{tabular}{|c|c|c|c|c|c|c|c|}
\hline \multirow{2}{*}{$\begin{array}{l}\text { Categoría } \\
\text { HOMA-IR }\end{array}$} & \multicolumn{2}{|c|}{ Obesos } & \multicolumn{2}{|c|}{ No obesos } & \multicolumn{2}{|c|}{ Total } & \multirow[t]{2}{*}{$p$} \\
\hline & $n$ & $\%$ & $n$ & $\%$ & $n$ & $\%$ & \\
\hline 1 (<33 percentil) & 7 & 20,6 & 16 & 45,7 & 23 & 33,3 & $0,021^{*}$ \\
\hline 2 ( 33 - 66 percentil) & 10 & 29,4 & 13 & 37,1 & 23 & 33,3 & 0,494 \\
\hline 3 (> 66 percentil) & 17 & 50,0 & 6 & 17,1 & 23 & 33,3 & $0,002^{*}$ \\
\hline Total & 34 & 100,0 & 35 & 99,9 & 69 & 99,9 & \\
\hline
\end{tabular}

${ }^{*} p<0,05$.

Tabla 5. Presencia de antecedentes familiares de riesgo de enfermedad cardiovascular en los grupos de estudio.

\begin{tabular}{lccc}
\hline Antecedente familiar & P ( Fisher ) & OR & 95\% LC ( OR ) \\
\hline Padre con angina & 0,004 & 13,2 & $1,6-1,99$ \\
Padre obeso & 0,041 & 2,1 & $1,08-4,3$ \\
Abuelo hipertenso & 0,018 & 1,185 & $1,14-3,02$ \\
Abuelo con angina & 0,043 & 2,41 & $1,1-5,32$ \\
Tío diabético & 0,001 & 3,15 & $1,66-5,99$ \\
Tío obeso & 0,03 & 2,7 & $1,16-6,27$ \\
\hline
\end{tabular}

\section{DISCUSIÓN}

Los presentes hallazgos en adolescentes de ambos sexos confirman las comunicaciones existentes en el sentido de observarse una relación positiva entre la resistencia a la insulina y la presencia de obesidad. Si bien los valores, empleando el HOMA-IR, suelen estar entre 0 y 15 , no existen puntos de corte claramente establecidos para la población pediátrica. Sin embargo, Weiss y col, en una investigación ${ }^{(27)}$ con adolescentes de edades promedio similares al presente estudio, refieren valores que se incrementan progresivamente en función al exceso de peso. Así, los pacientes sin sobrepeso tienen valores menores a 3 , mientras que los obesos presentan cifras mayores a 6 , hallazgo similar a la presente comunicación, donde el valor máximo encontrado en el grupo sin obesidad fue 4,3. También, podemos anotar que, a pesar de que ninguno de los escolares llegó al rango de obesidad severa (IMC mayor de 2,5 desviaciones estándar), se observó cifras de HOMAIR de hasta 12,5 en dicho grupo, algo mayor a lo encontrado en el estudio de Weiss, aunque comparable con el grupo de población hispánica (semejante a la nuestra). Esta situación fue corroborada con el hallazgo de que $50 \%$ de obesos tenía un HOMA-IR en rangos mayores al 66 percentil. Sin embargo, el no haber encontrado diferencias en el rango de 33 a 66 percentil (grupo 2), evidencia el carácter multifactorial que influye el comportamiento de la insulina, entre los que destacan los factores genéticos y la distribución corporal del tejido graso.

Además, es necesario señalar la relación de obesidad y resistencia a la Insulina con marcadores de riesgo cardiovascular, como la proteína $\mathrm{C}$ reactiva (PCR), en adolescentes obesos y con sobrepeso, según lo encontrado por Pajuelo y col (28), lo cual enfatiza la importancia de intervenciones nutricionales oportunas.

Por otra parte, es conocida la relación entre la resistencia a la insulina y la pubertad, la cual resulta en hiperinsulinemia, según refiere Arslanian ${ }^{(29)}$, fenómeno observado entre los estadios 2 y 4 de Tanner. Una posible explicación estaría en la mayor producción de hormona de crecimiento durante la pubertad, que 
disminuye la sensibilidad a la insulina, la misma que declina al llegar al final de la misma. Sin embargo, llama la atención que en el estadio 3 de Tanner de nuestro grupo de estudio se encuentre no solo una menor resistencia sino también una menor producción de insulina.

La homeostasis de la glucosa depende del balance entre la secreción de insulina por las células pancreáticas y la acción de la insulina. La identificación de un incremento en la función de la célula beta mediante el HOMA-\%B en los adolescentes obesos podría indicar un posible riesgo de desarrollar diabetes en el futuro, sabiendo que este incremento, unido a una disminución de la sensibilidad a la acción de la insulina, constituye la etapa que precede al agotamiento de la célula beta y la presentación clínica de la enfermedad (17).

Finalmente, el hallazgo de $43 \%$ de adolescentes obesos con dislipidemia y $71,8 \%$ con antecedente familiar de enfermedad cardiovascular u obesidad, asociado a la menor sensibilidad insulínica, muestra que es posible identificar a los sujetos en riesgo de continuar con alteraciones metabólicas en la vida adulta, con la finalidad de incorporarlos a un programa de atención integral que permita orientarlos a un estilo de vida saludable.

A pesar de que el HOMA-IR y el HOMA-\%B han sido empleados con mayor frecuencia para estudios en pacientes diabéticos adultos, según lo refiere Wallace ${ }^{(30)}$, los hallazgos del presente trabajo sugerirían su utilidad en la población pediátrica. Se recomienda realizar el estudio con un mayor número de adolescentes.

\section{REFERENCIAS BIBLIOGRÁFICAS}

1. Pinhas-Hamiel O, Dolan LM, Daniels SR, Standiford D, Khoury PR, Zeitler P. Increased incidence of non-insulin dependent diabetes mellitus among adolescents. J Pediat. 1996;128:608-15.

2. American Diabetes Association. Type 2 diabetes in children and adolescents. Pediatrics. 2002;105:671-80.

3. Amigo H. Obesidad del niño en América Latina: situación, criterios diagnósticos y desafíos. Cad Saúde Pública. 2003;19(Supl. 1):S163-70.
4. Pajuelo J, Rocca J, Gamarra M. Obesidad infantil: sus características antropométricas y bioquímicas. An Fac med. 2003;64(1):21-6.

5. Tershakovec AM, Koppler KM, Zemell BS, Katz I, Weinzimer S, Harty MP, et al. Body composition and metabolic factors in obese children and adolescents. Int J Obes. 2003;7:19-24.

6. Polonsky KS, Sturis J, Bell Gl. Non-insulin-dependent diabetes mellitus -a genetically programmed failure of the beta cell to compensate for insulin resistance. N Engl J Med. 1996;334:777-83.

7. Edelstein SL, Knowler WC, Bain RP, Andres R, Barrett-Connor EL, Dowse GK, et al. Predictors of progression from impaired glucose tolerance to NIDDM : an analysis of six prospective studies. Diabetes 1997; 46:701-10.

8. Haffner SM, Stern MP, Hazuda HP, Mitchell BD, Patterson JK. Cardiovascular risk factors in confirmed prediabetic individuals: does the clock for coronary heart disease star ticking before the onset of clinical diabetes. JAMA. 1990;263:2893-9.

9. Tuomilehto J, Lindstrom J, Ericksson JG, Valle TT, Hämäläinen $\mathrm{H}$, llanne-Parikka $\mathrm{P}$, et al. Prevention of type 2 diabetes mellitus by changes in lifestyle among subjects with impaired glucose tolerance. N Engl J Med. 2001;344:1343-50.

10. Reaven GM. Role of insulin resistance in human disease. Diabetes. 1988;37:1595-607.

11. Ferrannini E, Haffner SM, Mitchell BD, Stern MP. Hyperinsulinemia: the key feature of a cardiovascular and metabolic syndrome. Diabetologia. 1991;3:416-22.

12. Pajuelo J, Mosquera Z, Quiroz R. El sobrepeso y obesidad en adolescentes. Diagnóstico. 2003;42(1):17-22.

13. Argente J, Barrios V, Pozo J, Muñoz MT, Hervás F, Stene M, et al. Normative data for insulin-like growrth factors (IGFs), IGF-binding proteins, and growth hormone-binding protein in a healthy spanish pediatric population: age-. and sex.-related changes. J Clin End Metab. 1993;77(6):1522-8.

14. Copeland KC, Colletti RB, Devlin JT, McAuligge $\mathrm{TL}$. The relationship between insulin-like growth factor-I adiposity, and aging. Metabolism. 1990;39:584-7.

15. Crist DM, Hill JM. Diet and insulin-like growth factor I in relation to body composition in women with exercise-induced hypothalamic amenorrhea. J Am Coll Nutr. 1990;2:200-4.

16. Rosskamp R, Becker M, Soetadji S. Circulating somatomedin-C levels and the effect of growth hormone-releasing factor on the plasma levels of growth hormone the somatostatin-like inmunoreactivity in obese children. Eur J Pediatric. 1987;146:48-50.

17. Caprio S, Bronson M, Sherwin RS, Rife F, Tamborlane WV. Coexistence of severe insulin resistance, and hyperinsulinemia in preadolescent obese children. Diabetologia. 1996;39:1489-97.
18. Rosenbloom AL, Joe JR, Young RS, Winter WE. Emerging epidemic of type 2 diabetes in youth. Diabetes Care. 199;22(2):345-54.

19. Sinha R, Fisch G,Teague B, Tamborlane W, Banyas $B$, Allen $\mathrm{K}$, et al. Prevalence of impaired glucose tolerance among children and adolescents with marked obesity. N Engl J Med. 2002;346(11):80210.

20. Matthews DR, Hosker JP, Rudenski AS, Naylor BA, Treacher DF, Turner RC. Homeostasis model assessment: insulin resistance and beta-cell function from fasting plasma glucose and insulin concentrations in man. Diabetologia. 1985;28(7):412-9.

21. Yanovski J, Sovik KN, Sebring NG. Insulin-like growth factors and bone mineral density in African American and white girls. J Ped. 2000;137:82632.

22. Kahn BB, Flier JS. Obesity and insulin resistance. J Clin Invest. 2000;106:473-81.

23. Maffeis C, Moghetti P, Grezzani A, Clementi M, Gaudino R, Tato L. Insulin resistance an the persistence of obesity from childhood into adulthood. J Clin Endocrinol Metab. 2002;87(1):71-6.

24. Marshall WA, Tanner JM. Variations in patterns of pubertal changes in girls. Arch Dis Childhood. 1969;44:291-303.

25. Marshall WA, Tanner JM. Variations in the pattern of pubertal changes in boys. Arch Dis Childhood. 1970;45:13-23.

26. Must A, Jackes PF, Dallal GE, Bajema CY, Dietz WH. Long-term morbidity and mortality of overweight adolescents. N Engl J Med. 1992;327:1350-5.

27. Weiss R, Dziura J, Burgert TS, Tamborlane WV, Taksali SE, Yeckel CW, et al. Obesity and the metabolic syndrome in children and adolescents. N Engl J Med. 2004;350(23):2363-74.

28. Pajuelo J, Bernui I, Rocca J, Torres L, Soto L. Marcadores bioquímicos de riesgo cardiovascular en una población adolescente femenina con sobrepeso y Obesidad. Ann Fac med. 2009;70(1):7-10.

29. Arslanian SA, Kalhan SC. Correlations between fatty acid and glucose metabolism to entail explanation of insulin resistance of puberty. Diabetes. 1994;43:908-14.

30. Wallace TM, Levy JC, Matthews DR. Use and abuse of HOMA modeling. Diabetes Care. 2004;27:148795.

Manuscrito recibido el 16 de noviembre de 2009 y aceptado para publicación el 25 de febrero de 2010.

\section{Correspondencia}

Dr. Carlos Manuel Del Aguila Villar

Servicio de Endocrinología, Instituto Nacional de

Salud del Niño. Av. Brasil 600

Lima 5, Perú

Correo-e: caguilav@hotmail.com 